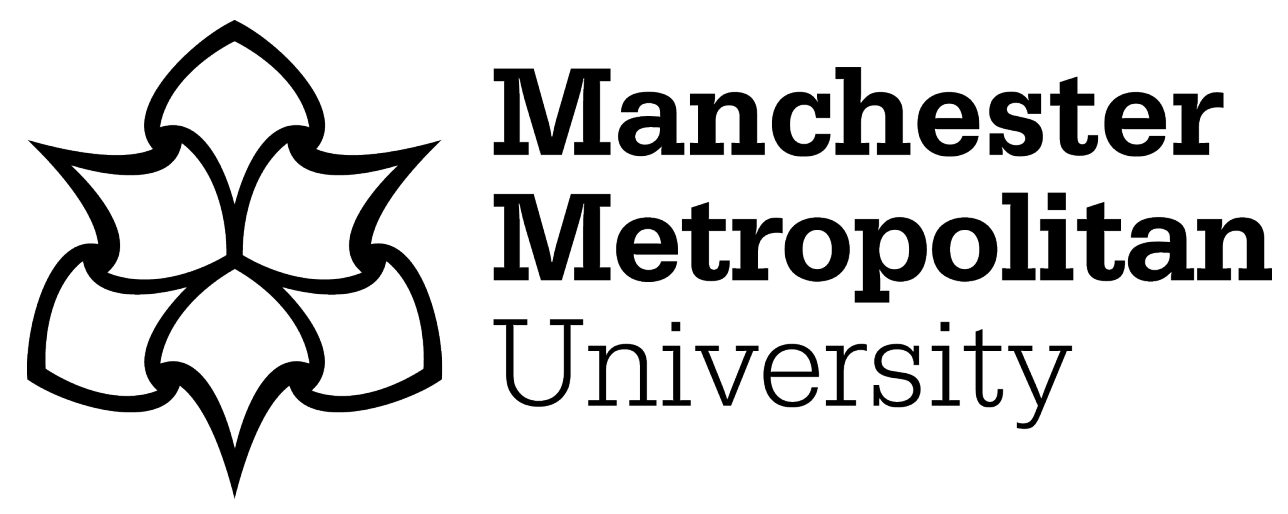

Anoh, K, Adebisi, B, Jogunlola, O and Hammoudeh, M (2017) Cooperative hybrid wireless-powerline channel transmission for peer-to-peer energy trading and sharing system. In: International Conference on Future Networks and Distributed Systems (ICFNDS 2017), 19 July 2017 - 20 July 2017, Cambridge, United Kingdom.

Downloaded from: https://e-space.mmu.ac.uk/620074/

Publisher: Association for Computing Machinery (ACM)

DOI: https://doi.org/10.1145/3102304.3102311

Please cite the published version 


\section{Cooperative Hybrid Wireless-Powerline Channel Transmission for Peer-to-Peer Energy Trading and Sharing System}

\author{
Kelvin Anoh \\ School of Engineering \\ Manchester metropolitan University \\ Manchester, UK \\ k.anoh@mmu.ac.uk \\ Olamide Jogunlola \\ School of Computing \\ Manchester metropolitan University, Manchester, UK \\ olamidel.jogunola@stu.mmu.ac.uk
}

\author{
Bamidele Adebisi \\ School of Engineering \\ Manchester metropolitan University \\ Manchester, UK \\ b.adebisi@mmu.ac.uk
}

Mohammad Hammoudeh

School of Computing

Manchester metropolitan University, Manchester, UK m.hammoudeh@mmu.ac.uk

\section{INTRODUCTION}

The early transceiver narrow-band (NB) powerline communication (PLC) models were deployed using single carrier modulation [21]. These NB-PLC systems are outdoor communication systems operating at $3-500 \mathrm{kHz}$ frequencies (e.g. PRIME, G3, IEEE 1901.2, ITU G.hnem); other standards operating within $500 \mathrm{kHz}-100 \mathrm{MHz}$ are broadbands (BB) and are usually deployed indoors (e.g. IEEE 1901 Home-plug, ITU-T G.hn) [8, 21, 22]. There are also the ultra-NBs (UNB) which operate within $125-3000 \mathrm{~Hz}$ [8]. Typical PLC channels of the smart-grid are equipped with smart meters which operate in the NB outdoor communication frequency of PLC systems.

Among the three PLC communication standard groups, the first standards to deploy multicarrier modulation is PRIME in 2007 [21] due to the fact the multicarrier modulation is more robust over impulsive fading channels. Like in IEEE 1901 [17], PLC system are designed using discrete Fourier transform (DFT) $[4,28]$ or wavelets $[5,6]$. OFDM multicarrier transmission achieves better bit error ratio (BER) performance than single-carrier in PLC systems because it can spread the impulsive noise (IN) over multiple symbols when data symbols are processed using the DFT [9, 20]. With OFDM, cyclic prefix $(\mathrm{CP})$ is used to combat intersymbol interference.

Conventionally, smart meters are designed to operate wirelessly or by using powerline or to switch between the communication standards [26]. To enhance performance, these independent fading channel and communication standards can be combined to operate in unison [16]. This involves transmitting the same signal over different fading channels and using maximal ratio combining (MRC) at the receiver in the recovery of the transmitted signal $[2,13]$. The ideal of this scheme follows from the fact that if one channel is severely degraded, the other may not be similarly impaired.

Since the peer-to-peer (P2P) energy trading and sharing (ETS) derives from the present smart-grid system [7, 19], we consider the integration of the hybrid wireless-powerline communication strategy, in cooperation, into the P2P-ETS future system. In P2PETS, the conventional passive consumers become active prosumers who generate, sell, share, buy and manage own energy generation. Sometimes called the energy internet [19], the participating peers pursue own selfish and independent goal which is generally aligned with the global energy system and community interests of lowering the carbon footprints. These active prosumers that

Permission to make digital or hard copies of all or part of this work for personal o classroom use is granted without fee provided that copies are not made or distributed for profit or commercial advantage and that copies bear this notice and the full citation on the first page. Copyrights for components of this work owned by others than ACM must be honored. Abstracting with credit is permitted. To copy otherwise, or republish, to post on servers or to redistribute to lists, requires prior specific permission and/or a fee. Request permissions from permissions@acm.org.

ICFNDS '17, Cambridge, United Kingdom

(C) 2017 ACM. 978-1-4503-4844-7/17/07 ..\$15.00

DOI: $10.1145 / 3102304.3102311$ 


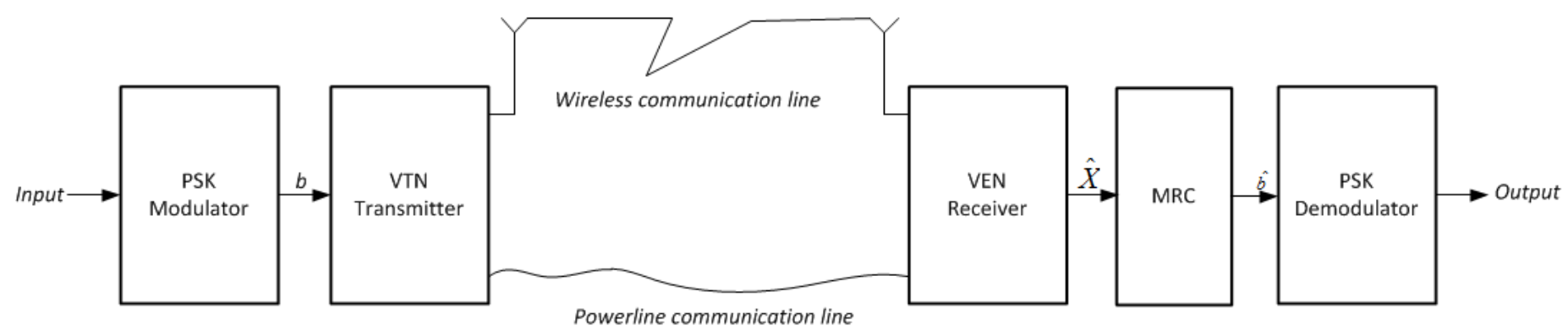

Figure 1: System model of the proposed cooperative wireless-powerline channel communication

generate, store and sell energy can communicate with another participating peer withing the P2P-ETS community through either the wireless or powerline systems. In this study, we consider a system of communication that cooperatively harnesses the strengths of both communication strategies to ensure resilient communications among the P2P-ETS community. A closely related technology, namely openADR, enables the demand-side energy management through virtual terminal node (VTN) and virtual end node (VEN). Although most studies in the literature consider the networking level and below such as in the openADR standard [1, 11, 12, 14], except for the cross-layer investigation in [21], this study is the foremost to the best of our knowledge that investigates the physical layer architecture of the VTN and VEN deployment with respect to P2P-ETS.

Traditionally, VTNs, VTN/VENs and VEN (end-users) are actors of the conventional openADR system. During a communication the VTN sends information to the VENs; wherein there are many other VENs, one VEN can be selected to operate as a VTN overseeing communications to the rest VENs, hence VTN/VEN. Since the market system uses powerline, we exploit the energy transmission cables as a suitable channel to reach a peer within the P2P-ETS community. For simplicity, we shall consider only one VTN to one VEN (VTN-VEN) communication, which can as well be extended to any other form of communication arrangements within the ETS community.

In $[22-24,26]$ the authors illustrated an example of hybrid wirelesspowerline communication in case of smart-metering; this allows only a half-duplex transmission (for example from utility to the data-sink/company database). However, in VTN-VEN communication in the default openADR case, the VTN triggers the request and the VEN responds. When fully matured, the openADR model will then involve a full-duplex transmission such as in the P2PETS communication strategy. In terms of capacity, the cooperative wireless-PLC system achieves better performance than when solely operating either wireless or PLC standard [10]. Similarly, the hybrid channel communication achieves increased BER performance [13].

In this study, we suggest the use of singular value decomposition (SVD) method to maximize the received signal power at the receiver to further enhance BER performance. Thus, at the transmitter we proposed a precoded signal transmission over hybrid wireless-powerline channel involving two communicating P2P-ETS peers. This proposal finds application into the $\mathrm{P} 2 \mathrm{P}$ communication during, for example, energy trading and sharing. We model the wireless channel under the Rayleigh fading distribution assumption while the powerline model is modeled using the log-normal fading distribution. Then at the receiver, we detect the transmitted signal estimate using the conjugate transpose of the eigenvector of the channel matrix. This method requires that perfect knowledge of the channel state information (CSI) at the transmitter. The problem formulation is presented in Section 2 while the method SVD formation including transmit signal precoding is described in Section 3. Our results are presented in Section 4 with the conclusion following.

\section{PROBLEM FORMULATION}

In this work, we consider a hybrid wireless and powerline channels operating in cooperation. In other words, the transmitted signal from the wireless channel is as required as the signal communicated over a powerline channel. A simplified high-level architecture of the system model under study is as shown in Fig. 1. The input data could involve digitized energy prices, for example, modulated using binary phase-shift keying (BPSK) and converted to OFDM time signals as

$$
s(n)=\frac{1}{\sqrt{N}} \sum_{k=0}^{N-1} X(k) \exp \left(j 2 \pi \frac{n k}{N}\right) \forall n=0,1, \cdots, N-1 .
$$

where $X(k)$ is the digitized frequency domain input signal. The output time domain signal can be transmitted over powerline or wireless channel as shown in Fig. 1 of a VTN gateway. Now, considering the signals over the wireless and powerline channels respectively, then

$$
\begin{aligned}
x_{w} & =h_{w} s_{w}+z_{w} \\
x_{p} & =h_{p} s_{p}+z_{p}
\end{aligned}
$$

where $x_{w}$ is the received signal over wireless channel and $x_{p}$ is the received signal from the powerline channel. $s_{w}$ and $s_{p}$ are the original transmitted signals over wireless and PLC channels respectively. In addition, $h_{w}$ is the impulse response of the wireless channel which we model as Rayleigh distributed with zero mean and variance $\sigma_{h_{w}}^{2}$ while $h_{p}$ is the impulse response of the powerline channel which we model as log-normal distributed. The wireless channel model can be represented from the Rayleigh multipath fading model with the probability density function (PDF) of the form

$$
f_{\left|h_{w}\right|}\left(h_{w} ; \mu_{h_{w}}, \sigma_{h_{w}}\right)=\frac{1}{\sigma_{h_{w}} \sqrt{2 \pi}} \exp \left(-\frac{1}{2}\left(\frac{\left|h_{w}\right|-\mu_{h_{w}}}{\sigma_{h_{w}}}\right)^{2}\right)
$$


where $\mu_{h_{w}}$ is the mean, $\sigma_{h_{w}}$ is the standard deviation and $\sigma_{h_{w}}^{2}$ is the variance of $h_{w}$. In addition, we model the powerline channel model as obeying the log-normal fading distribution with PDF as

$$
f_{\left|h_{p}\right|}\left(h_{p} ; \mu_{h_{p}}, \sigma_{h_{p}}\right)=\frac{1}{h_{p} \sqrt{2 \pi \sigma_{h_{p}}^{2}}} e^{\left(-\left(\frac{\ln \left(h_{p}\right)-\mu_{h_{p}}}{\sqrt{2 \sigma_{h_{p}}^{2}}}\right)^{2}\right)}
$$

where $\mu_{h_{p}}$ is the mean, $\sigma_{h_{p}}$ is the standard deviation and $\sigma_{h_{p}}^{2}$ is the variance of Gaussian distributed random variable, $\ln \left(h_{p}\right)$. To avoid attenuation or amplifying the average received power, the mean power of the fading channel must be normalized to unity such as $\mathbb{E}\left\{|h|^{2}\right\}=1$ for both links [13], where $\mathbb{E}\{\cdot\}$ is the statistical expectation value operator. Generally, the non-Gaussian noise part of a PLC channel route can be described as

$$
z_{p}=z_{w}(n)+z_{i}(n) \forall n=0,1, \cdots, N-1
$$

where $z_{w}$ is the conventional additive white Gaussian noise (AWGN) and $z_{i}(n)$ is the impulsive noise (IN) which can be expressed as [27]

$$
z_{i}(n)=b(n) \cdot \boldsymbol{n}_{w}(n), \forall n=0,1, \cdots, N-1
$$

where $\boldsymbol{n}_{w}(n) \sim \mathcal{N}\left(0, \frac{N_{0}}{2} \Gamma\right), \forall n=0,1, \cdots, N-1$ and $b(n)$ is the Bernoulli process that can be expressed as

$$
\operatorname{Pr}\{b(n)\}=\left\{\begin{array}{ll}
p, & b(n)=1 \\
0 & b(n)=0
\end{array}, \forall n=0,1, \cdots, N-1\right.
$$

where $N_{0}$ is the single-sided power spectral density and $\Gamma$ is the mean power ratio of the IN and AWGN components. Considering an mixture IN-Gaussian noise channel with Gaussian noise part as $z_{w} \sim \mathcal{N}\left(0, \sigma_{w}^{2}\right)$ and $z_{i} \sim \mathcal{N}\left(0, \sigma_{i}^{2}\right)$ is the IN part where $\sigma_{w}^{2}$ and $\sigma_{i}^{2}$ are AWGN and IN noise variances. The PDF of the powerline system can be expressed as [18]

$$
f_{z}\left(z ; \mu_{z}, \sigma_{z}\right)=\sum_{l=0}^{L=1} p_{l} \mathcal{N}\left(z_{0}(n) ; 0, \sigma_{z, l}^{2}\right) \forall n=0,1, \cdots, N-1
$$

where $\mathcal{N}\left(z_{0} ; 0, \sigma_{z, l}^{2}\right)=\frac{1}{\sigma_{z, l} \sqrt{2 \pi}} \exp \left(-\frac{1}{2}\left(\frac{z_{0}-\mu_{z}}{\sigma_{z, l}}\right)^{2}\right)$ is the Gaussian PDF of $z(n)$ with $z_{0}$ discrete envelope, zero-mean $\left(\mu_{z}=0\right)$, variance $\sigma_{z, l}^{2}$ and $p_{l}$ is the mixing probability of the $l^{\text {th }}$ component.

From combining (2a) and (2b), the received signal with IN and AWGN over the two fading channels (in cooperation) can be expressed as

$$
X=H S+Z
$$

where the characteristics components of the received signal can be explicitly decomposed into the following

$$
\begin{gathered}
\boldsymbol{H}=\left[\begin{array}{cc}
h_{w} & 0 \\
0 & h_{p}
\end{array}\right] \\
S(n)=\left[\begin{array}{c}
s_{w} \\
s_{p}
\end{array}\right], \forall n=0,2, \cdots, N-1 \\
Z=\left[\begin{array}{c}
z_{w} \\
z_{p}
\end{array}\right] .
\end{gathered}
$$

From (10), the noise vector is a hybrid wireless and powerline model. While $z_{w}$ is Gaussian distributed, $z_{p}$ is a mixture of Gaussian and
IN. Notice that the transmitted signal as described by [13] are representatively uncorrelated. In [2], we showed that transmitting the same message that are precoded over different eigen-fading channels achieve better BER performance than transmitting different signals.

From (10b), $s_{p}, s_{w} \in d$, where $d(n), \forall n=0,1, \cdots, N-1$ are the BPSK modulated OFDM signal from (1). This architecture can enhance BER performance [2]. For example, the messages to be sent over the wireless and PLC different fading channel are the same message, such that if the wireless fading channel is badly faded, the powerline channel may not be similarly faded and vice versa. We give consideration only to the use of maximum ratio combining (MRC) receiver in the hybrid wireless-powerline model as it has been shown in $[2,23,24,26]$ to outperform the selection combining approach. Thus, we rewrite (10b) as

$$
S(n)=\left[\begin{array}{c}
s_{w} \\
s_{p}
\end{array}\right]=I_{n_{t}} \otimes s(n) \forall n=0, \cdots, N-1,
$$

where $\otimes$ is the Kronecker product, $n_{t}=2$ is the number of transmitting branches and $I_{n_{t}}$ is an identity matrix of $n_{t}$ dimension. It follows that $\forall n=0, \cdots, N-1, s_{p}(n)=s_{w}(n)$ at the transmitter.

\section{RECEIVED SIGNAL DETECTION AT THE RECEIVER}

We present three different models for performing communications over the PLC fading channel. These are described to harness the best performance of the hybrid wireless-powerline channel model over cooperative channel transmission in a P2P system of energy internet. Meanwhile, let us express the simplified general received signal at the receiver after converting to frequency domain as

$$
Y=\rho H S+Z
$$

where $\rho$ is a scaling factor that ensures equal energy dissipation on each transmission branch, $S \in \mathbb{C}^{n_{t} \times 1}$ is the input signal vector, $Z \in \mathbb{C}^{n_{r} \times 1}$ is the non-Gaussian noise vector and $H \in \mathbb{C}^{n_{r} \times n_{t}}$ is the hybrid wireless-powerline channel matrix model which can be further decomposed into wireless-powerline component parts as $h_{w} \in H$ and $h_{p} \in H$ with the distributions $h_{w} \sim C \mathcal{N}\left(0, \sigma_{h_{w}}^{2}\right)$ and $h_{p} \sim \mathcal{C N}\left(\mu_{h_{p}}, \sigma_{h_{p}}^{2}\right)$. We can then describe the signal detection in three ways. For example, considering zero-forcing (ZF) model, the received signal can be represented as

$$
\hat{s}(n)=\rho s(n)+\frac{1}{H(n)} Z(n)
$$

From (13), the hybrid channel matrix amplifies the combined AWGN and IN - this will degrade the BER performance of the system. One of the ways of minimizing the noise overhead during signal detection is by applying this is by applying MMSE equalization style as

$$
\hat{s}=\frac{H^{*} X}{|H|^{2}+\left(\frac{E_{s}}{N_{0}}\right)^{-1}}=\left(\rho s+\frac{H^{*}}{|H|^{2}+\left(\frac{E_{s}}{N_{0}}\right)^{-1}} Z\right)
$$

where $(\cdot)^{*}$ represents the complex conjugate of $(\cdot)$ and $E_{s} / N_{0}$ is the received signal to noise power. In addition, the mutual channel 
interference can be minimized by maximizing the channel gain index using SVD, such as $[2,3]$

$$
H=U \lambda V^{\mathcal{H}}
$$

where $U \in \mathbb{C}^{n_{t} \times n_{t}}$ and $V \in \mathbb{C}^{n_{t} \times n_{t}}$ are unitary matrices such that $U^{\mathcal{H}} U=I_{n_{t} \times n_{t}}$ and also $V^{\mathcal{H}} V=I_{n_{t} \times n_{t}}$. In Section 1, we stated that the CSI is perfectly known at the transmitter and receiver. Thus, we can precode the original input signal, $S$, such as

$$
C=V \times S
$$

where $C \in \mathbb{C}^{1 \times n_{t}}$ and $V$ is a vector of the eigen-decomposition of the channel which we model as flat fading channel model. By combining the precoded signal in (16) at the transmitter and the received signal (12), then the received signal in terms of the SVD processing can be expressed as

$$
\begin{aligned}
\hat{\boldsymbol{s}} & =U^{\mathcal{H}} X \\
& =U^{\mathcal{H}} H C+U^{\mathcal{H}} \mathcal{Z} \\
& =U^{\mathcal{H}}\left(U \lambda V^{\mathcal{H}}\right) C+U^{\mathcal{H}} \mathcal{Z} \\
& =U^{\mathcal{H}}\left(U \lambda V^{\mathcal{H}}\right) V S+U^{\mathcal{H}} \mathcal{Z} \\
& =\lambda S+U^{\mathcal{H}} \mathcal{Z}
\end{aligned}
$$

where $\lambda=\sigma_{1} \leq \sigma_{2} \leq \cdots \leq \sigma_{r}$ are positive semi-definitive values for a matrix with rank, $r$ and $\sigma_{m} \forall m=1, \cdots, r$ is the gain allocated to each of the fading channel sources. Obviously, $r=2$ for the channel matrix in (10a). If $\hat{\mathcal{Z}}=U^{\mathcal{H}} \mathcal{Z}$, both $\mathcal{Z}$ and $\hat{\mathcal{Z}}$ have the same statistical property since the distribution of $\mathcal{Z}$ is invariant under unitary transformation [25]. In terms of throughput, practical measurements campaign for different methods of combining hybrid wireless-powerline communications has shown that MRC combing scheme at the receivers achieves the highest throughput among optimum combining state known (OCSK), best link combining (BLC), saturated metric combining (SMC) and MRC [15]. The MRC optimal performance is analytically and using simulations corroborated in [13]. Compared to the results reported in [21], the cooperation model coupled with SVD detection method at the receiver will greatly enhance the output result as will be shown in Section 4

\section{RESULTS AND DISCUSSION}

Supposing a conventional openADR system equipped with one VTN facility of the ISO utility (in other words, the transmitter), and one VEN (receiver) facility, also equipped with the hybrid wireless and powerline standards described in Section 1 of this paper at both nodes. We are aware, however, that the present openADR system do not support P2P communication. Meanwhile, the hypothetical architecture provides platform for improvement and incorporation of new performing strategy and standards to enhance the reliability of the system. The VTN modulates energy data of an ETS-P2P prosumer using BPSK, then passes it through an IFFT block to generate the time domain signal and CP appended. This is passed over a non-Gaussian PLC channel with IN and the log-normal fading of the PLC system. Also, the same same is passed through the wireless channel to enable multiple signal reception at the receiver.

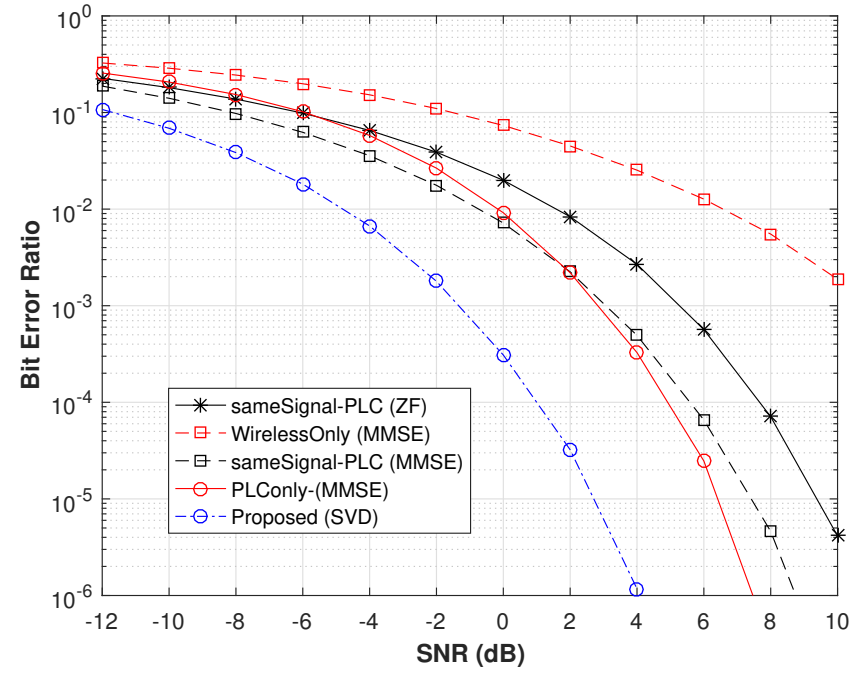

Figure 2: Performance comparison of proposed cooperative transmission and conventional multi-channel communication with and without signal precoding, $p=10^{-2}$, BPSK, $N=256$

At the receiver, we remove $\mathrm{CP}$ and transform the received signal back into the frequency domain. Here, frequency domain domain $\mathrm{ZF}$ and MMSE are performed. Since receiver is equipped with multichannel reception, the received signals are combined using MRC and passed through BPSK demodulator whence error calculation is performed afterwards. In the case of SVD model, we assume that the CSI is known both to the transmitter and the receiver. Thus, we compute the SVD of the channel matrix and use $V$ to precode the transmitting signal and $U^{\mathcal{H}}$ to equalize the channel at the receiver. From (17), the SVD maximizes the system performance with $\lambda$ gain induced to the output signal. The property is absent in ZF and MMSE.

In Fig. 2, the results of hybrid transmission detected using ZF, MMSE and SVD are presented. Alongside, the results of solely wireless and solely powerline transmissions are shown. We see that the wireless transmission clutches the poorest performance among all schemes due to wireless multipath fading. With MMSE, better BER is achieved as it minimizes the noise effects better than ZF. At low SNR, the hybrid wireless-powerline system performs better than both solely wireless or powerline system, but slightly less than the powerline at increased SNR. In all however, the SVD with precoding significantly outperforms the hybrid MMSE-detected wireless-powerline system, for example by $2.2 \mathrm{~dB}$ gain and also 1.5 $\mathrm{dB}$ better than transmitting over solely powerline. Notice also that by applying MMSE clearly outperforms ZF by $1 \mathrm{~dB}$.

Afterwards, we increased the probability of impulsive noise occurrence by $10 \%$ (i.e. from $p=10^{-2}$ to $p=10^{-1}$ ). We apply MMSE to enhance performance by minimizing the error floor introduced by the ZF noise overhead. For example, we detect the received signal using MMSE. The MMSE minimizes the error floor introduced by compensating the channel at the receiver. We find that the BER 


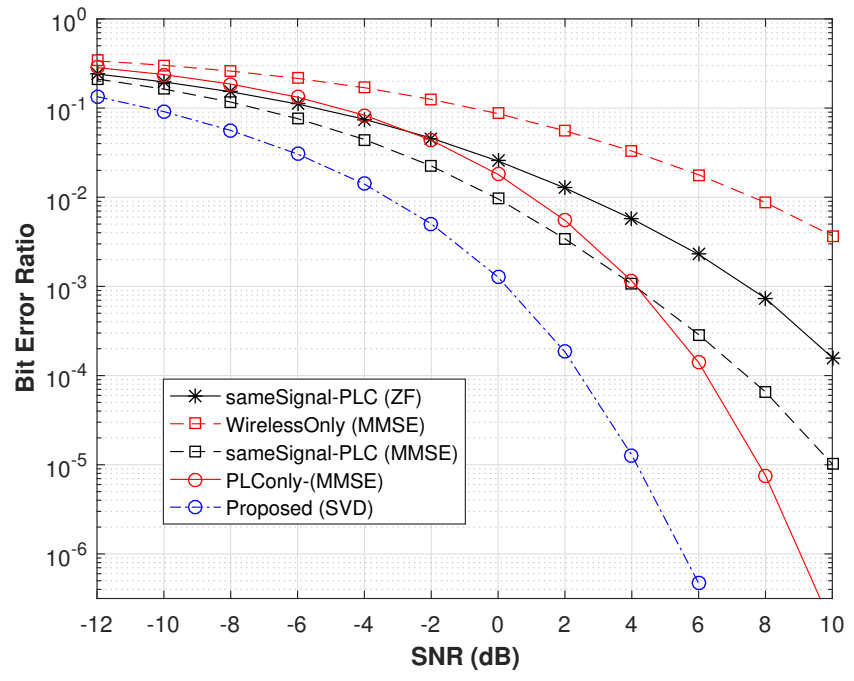

Figure 3: Performance comparison of proposed cooperative transmission and conventional multi-channel communication with and without signal precoding, $p=10^{-1}$, BPSK, $N=256$

is reduced by increasing the probability of impulsive noise occurrence; this is expected due to the excess noise power corrupting the originally transmitted signal. However, Fig. 3 shows that the SVD detection technique offers better signal reception at the receiver corroborating our earlier results in Fig. 2.

\section{CONCLUSION}

We have presented, in this study, the performance evaluation of a hybrid wireless-powerline system with cooperative signal detection at the receiver. The powerline channel model was modeled using the log-normal distribution while the wireless channel follow the Rayleigh fading distribution. We performed signal detection using zero-forcing, MMSE and conjugate of eigen-vector from singular value decomposition which we used to precode the signals. At the receiver, the signal received from each transmitting branch qualify for MRC combining when the noise variances of the wireless and powerline channels are relaxed to be Gaussian. Our results show that using the conjugate of eigen-vector from singular value decomposition to detect the cooperative wireless-powerline channel achieves the best BER performance. This result can find application beyond the present state-of-the-art openADR system and smart-metering of the conventional smart-grid network into the forthcoming peer-to-peer energy trading and sharing systems.

\section{REFERENCES}

[1] OpenADR Alliance. 2013. OpenADR 2.0 b profile specification. Raportti. Ope$n A D R$ Alliance (2013)

[2] Kelvin Anoh, Bamidele Adebisi, and Godfrey Okorafor. 2016. Precoding of Correlated Symbols for STBC Systems Design. In Intl. Conf. Wireless Satellite Syst. Springer, 83-91.

[3] K. Anoh, E. Elkazmi, R. Abd-Alhameed, O. Madubuko, M. Bin-Melha, S. Jones, and T. Ghazaany. 2013. Improved multi-antenna system capacity using beamformer weights. In 2013 8th IEEE Design and Test Symposium. 1-4. DOI : http://dx.doi. org/10.1109/IDT.2013.6727086
[4] Kelvin OO Anoh, Rameez Asif, Raed A Abd-Alhameed, Jonathan Rodriguez, James M Noras, Steve MR Jones, and Abubakar S Hussaini. 2014. Multi-Antenna OFDM System Using Coded Wavelet with Weighted Beamforming. Radioengineering 23, 1 (2014), 356-361.

[5] Kelvin OO Anoh, Trust T Mapoka, Raed A Abd-Alhameed, Ogechukuka Ochonogor, and Steve MR Jones. 2014. On the Application of Raised-Cosine Wavelets for Multicarrier Systems Design. IRECAP 4, 4 (Aug. 2014), 143-150.

[6] Kelvin OO Anoh, James M Noras, Raed A Abd-Alhameed, Steve MR Jones, and Konstantinos N Voudouris. 2014. A new approach for designing orthogonal wavelets for multicarrier applications. AEU-Intl. f. Electron. Commun. 68, 7 (Jul. 2014), 616-622.

[7] I Safak Bayram, Muhammad Z Shakir, Mohamed Abdallah, and Khalid Qaraqe. 2014. A survey on energy trading in smart grid. In , 2014 IEEE Global Conf. Signal and Info. Proces. (GlobalSIP). 258-262.

[8] Cristina Cano, Alberto Pittolo, David Malone, Lutz Lampe, Andrea M Tonello, and Anand G Dabak. 2016. State-of-the-art in Power Line Communications: from the Applications to the Medium. IEEE 7. Sel. Areas Commun. 34, 7 (Feb. 2016), 1935-1952.

[9] Huaiyu Dai and H Vincent Poor. 2003. Advanced signal processing for power line communications. IEEE Commun. Mag. 41, 5 (May 2003), 100-107.

[10] Victor Fernandes, Mateus L Filomeno, Weiler A Finamore, and Moisés V Ribeiro. 2016. An Investigation on Narrow Band PLC-Wireless Parallel Channel Capacity. XXXIV SIMPOSIO BRASILEIRO DE TELECOMUNICACOES (Aug. 30 - Sept. 02, 2016), $834-838$.

[11] Girish Ghatikar and Rolf Bienert. 2011. Smart grid standards and systems interoperability: a precedent with OpenADR. In proceedings of the Grid Interop Forum. $1-7$.

[12] Girish Ghatikar, Edward L Koch, and Jim Boch. 2012. OpenADR Advances. ASHRAE fournal 54, 11 (2012), B16.

[13] Sabih Güzelgöz, Hasan Basri Celebi, and Hüseyin Arsian. 2010. Analysis of a multi-channel receiver: Wireless and PLC reception. In 2010 18th European Signal Process. Conf. 1106-1110.

[14] Ulrich Herberg, Daisuke Mashima, Jorjeta G Jetcheva, and Sanam MirzazadBarijough. 2014. OpenADR 2.0 deployment architectures: Options and implications. In 2014 IEEE Intl. Conf. Smart Grid Commun. (SmartGridComm). 782-787.

[15] Stephen Lai, Geoffrey Messier, and Lutz Lampe. 2012. Wireless/Powerline Diversity Communication. ISPLC (2012).

[16] Stephen W Lai and Geoffrey G Messier. 2012. Using the wireless and PLC channels for diversity. IEEE Trans. Commun. 60, 12 (Dec. 2012), 3865-3875.

[17] Lutz Lampe, Andrea M Tonello, and Theo G Swart. 2016. Power Line Communications: Principles, Standards and Applications from multimedia to smart grid (second ed.). John Wiley \& Sons.

[18] Jing Lin, Marcel Nassar, and Brian L Evans. 2013. Impulsive Noise Mitigation in Powerline Communications Using Sparse Bayesian Learning. IEEE 7. Sel. Areas Commun. 31, 7 (Mar. 2013), 1172-1183.

[19] J. Lin, T. Pande, I. H. Kim, A. Batra, and B. L. Evans. 2017. A New Framework for Peer-to-Peer Energy Sharing and Coordination in the Energy Internet. In IEEE ICC 2017 Green Communications Systems and Networks Symposium. pp. DOI : http://dx.doi.org/10.1109/ICC.2015.7248412

[20] YH Ma, PL So, E Gunawan, and YL Guan. 2004. Modeling and analysis of the effect of impulsive noise on broadband PLC networks. In Proc. of ISPLC 2004. $45-50$.

[21] J. Matanza, S. Kiliccote, S. Alexandres, and C. Rodrguez-Morcillo. 2015. Simulation of low-voltage narrow-band power line communication networks to propagate OpenADR signals. F. Commun. Netw. 17, 6 (Dec. 2015), 656-664. DOI : http://dx.doi.org/10.1109/JCN.2015.000112

[22] Mostafa Sayed and Naofal Al-Dhahir. 2014. Narrowband-PLC/wireless diversity for Smart Grid communications. In 2014 IEEE GLOBECOM. 2966-2971.

[23] Mostafa Sayed, Ghadi Sebaali, Brian L Evans, and Naofal Al-Dhahir. 2015. Efficient diversity technique for hybrid narrowband-powerline/wireless smart grid communications. In 2015 IEEE Intl. Conf. SmartGridComm. 1-6.

[24] Mostafa Sayed, Theodoros A Tsiftsis, and Naofal Al-Dhahir. 2017. On the Diversity of Hybrid Narrowband-PLC/Wireless Communications for Smart Grids. IEEE Trans. Wireless Commun. (2017).

[25] Wael Abu Shehab and Zouhair Al-qudah. 2017. Singular Value Decomposition: Principles and Applications in Multiple Input Multiple Output Communication system. Intl. f. Comput. Netwo. Commun. 9, 1 (Jan. 2017), 13 - 21.

[26] Junmo Sung and Brian L Evans. 2017. Real-Time Testbed for Diversity in Powerline and Wireless Smart Grid Communications. arXiv preprint arXiv:1609.04135v2 [cs.IT] (Mar. 2017).

[27] Der-Feng Tseng, Yunghsiang S Han, Wai Ho Mow, Li-Chung Chang, and AJ Han Vinck. 2012. Robust clipping for OFDM transmissions over memoryless impulsive noise channels. IEEE Commun. Lett. 16, 7 (Jul. 2012), 1110-1113.

[28] S. Weinstein and P. Ebert. 1971. Data Transmission by Frequency-Division Multiplexing Using the Discrete Fourier Transform. IEEE Trans. Commun. Technol. 19, 5 (Oct. 1971), 628-634. DOI : http://dx.doi.org/10.1109/TCOM.1971.1090705 